\title{
Singularities and Similarities
}

\author{
J. G. Eggers \\ School of Mathematics, University of Bristol, \\ University Walk, Bristol BS8 1TW, \\ United Kingdom \\ Jens.Eggers@bris.ac.uk
}

\subsection{Introduction}

In this series of lectures, I discuss the mathematical description of phenomena which cover many different length or time scales. Namely, it is extremely common that parts of the solution "living" on different scales look identical under a rescaling of their dimensions: they are self-similar. Some of the reasoning that explains this observation is given below, but the ubiquity of such scaling phenomena remains remarkable. More pragmatically, self-similarity is the reason why many-scaled phenomena are amenable to a theoretical description in the first place. Simply put, it means they can be reduced to something that has only one scale, and the complexity is all in the transformation from one scale to the other.

The examples and illustrations given below are all taken from hydrodynamics, other examples (without much danger of overlap) could have been taken from completely different fields of mathematics, physics, or engineering. In particular, I focus on singularities, i.e. the development of arbitrarily small structures in finite time. One such example occurs when a fluid drop falls from a faucet: at some point the thickness of the fluid neck separating the drop from the faucet must go to zero. As the singularity is approached, smaller and smaller length scales are covered, until the scale of molecules is reached.

Many of the points and observations made below are discussed in much more detail in the beautiful book by Barenblatt (1996). I also describe an instance where strict self-similarity breaks down, and the concept needs to be generalized. Such generalizations are an important topic of current research. In the end I discuss drop breakup on a very small scale, such that thermal fluctuations become important: self-similarity can then only be valid in a suitably defined averaged sense. 


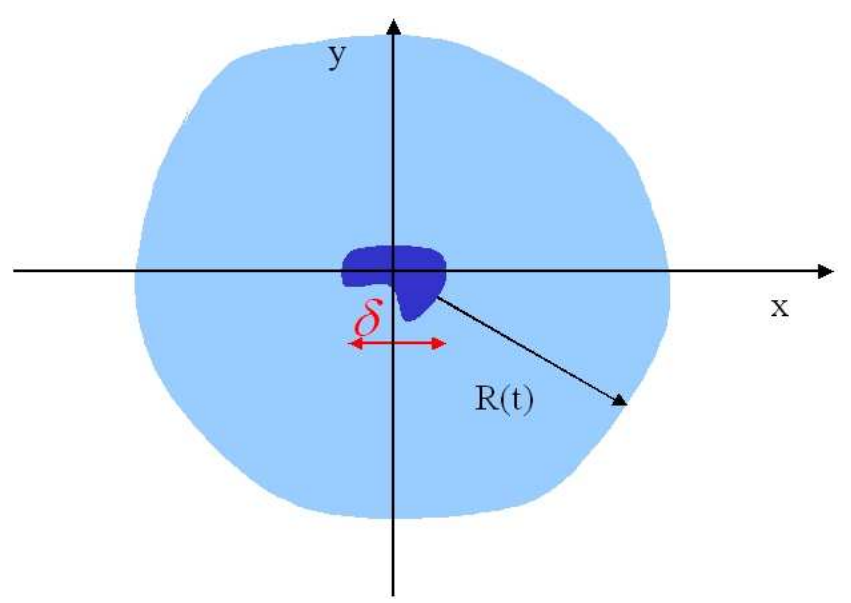

Figure 1: A spreading drop whose initial size was $\delta$ and of arbitrary shape.

\subsection{A simple example: the diffusion equation}

An initial drop of contaminant (typical size $\delta$ ) spreads diffusively in a container of site L. The spreading law is:

$$
\frac{\partial u}{\partial t}=\kappa\left(\frac{\partial^{2} u}{\partial x^{2}}+\frac{\partial^{2} u}{\partial y^{2}}\right) \equiv \kappa \Delta u .
$$

What does the solution for the contaminant density $u(x, t)$ look like? Plausibly the long-term solution can depend only on the amount of "stuff" $Q$, since this quantity is conserved during the evolution. From the initial value one obtains

$$
Q=\int_{\mathbb{R}^{2}} u(x, 0) d^{2} x
$$

Letting the dimension of $Q$ be mass, the dimensions of the other quantities become:

$$
[u]=\frac{g}{c^{2}},[\kappa]=\frac{\mathrm{cm}^{2}}{s},[x]=[y]=[\delta]=[L]=\mathrm{cm} .
$$

The most general form of the spreading law is

$$
u=f(\underbrace{Q, \kappa, t, x, y, \delta, L} . .),
$$

a mess!

$$
7 \text { arguments }
$$

First we use dimensional analysis, making everything dimensionless using characteristic length and mass scales in the problem. Namely: $\ell=\sqrt{\kappa t} \quad$ (involving the diffusion constant) and $u_{0}=Q / \ell^{2}$ :

$$
u=u_{0} F\left(\frac{x}{\ell}, \frac{y}{\ell}, \frac{\delta}{\ell}, \frac{L}{\ell}\right) .
$$

Since this result is invariant under the choice of 3 arbitrary dimensional scales $(\mathrm{cm}, \mathrm{s}, \mathrm{g})$ the number of arguments is now $4=7-3$. 
Second we use physical reasoning to further reduce the number of parameters. If the drop has spread to a much greater size than $\delta$, yet is still much smaller than the size of the box L: $\delta / \ell \ll 1, L / \ell \gg 1$, then the dynamics should have "forgotten" about the initial condition, but doesn't yet "feel" the outer boundary: $\mathrm{F}$ does not depend on $\delta / \ell$ or $L / \ell$. More formally: we replace the arguments by their limits and hope that $\mathrm{F}$ remains finite!

$$
u=u_{0} F\left(\frac{x}{\ell}, \frac{y}{\ell}, 0, \infty\right) \equiv u_{0} \bar{F}\left(\frac{x}{\ell}, \frac{y}{\ell}\right)
$$

We call

$$
u=\frac{Q}{\kappa t} \bar{F}\left(\frac{x}{\sqrt{\kappa t}}, \frac{y}{\sqrt{\kappa t}}\right)
$$

a similarity solution. A more general structure is

$$
u=f(t) F\left(\frac{x_{1}}{f_{1}(t)}, \frac{x_{2}}{f_{2}(t)}, \ldots\right)
$$

because a change in t only results in the rescaling of dependent and independent variables. In most cases $f_{i}(t)$ is a power law; below we are going to say a few words as to why power laws are special.

Now if $u$ has "forgotten" about the initial condition $u(\boldsymbol{x}, 0), \overline{\mathrm{F}}$ must depend on $x / \ell$ and $y / \ell$ in the same way: any anisotropy can only come in through the initial condition. Thus

$$
u=\frac{Q}{\kappa t} G\left(\frac{|\boldsymbol{x}|}{\sqrt{\kappa t}}\right), \quad \xi:=\frac{|\boldsymbol{x}|}{\sqrt{\kappa t}}
$$

is the form of the desired similarity solution.

Plugging this into the original equation, we find an equation for $G$ :

$$
-\frac{Q}{\kappa t^{2}} G-\frac{Q}{2 \kappa t} \frac{|x|}{\sqrt{\kappa} t^{3 / 2}} G^{\prime}=\frac{\kappa Q}{\kappa^{2} t^{2}} \frac{1}{\xi}\left(\xi G^{\prime}\right)^{\prime}, \quad\left[\text { note: } \Delta f(r)=\frac{1}{r}\left(r f^{\prime}\right)^{\prime}\right]
$$

hence

$$
-G^{\prime}-\frac{\xi}{2} G^{\prime}=\frac{1}{\xi}\left(\xi G^{\prime}\right),
$$

which is a called a similarity equation.

Equation (4) is easily solved:

$$
\begin{aligned}
& -\frac{1}{2}\left(\xi^{2} G^{\prime}\right)^{\prime}=\left(\xi G^{\prime}\right)^{\prime} \rightarrow-\frac{\xi^{2}}{2} G=\xi G^{\prime}+C, \text { put } \xi=0 \rightarrow C=0 \\
& G^{\prime}=-\frac{\xi G}{2} \rightarrow G=G_{0} e^{-\xi 2 / 4}
\end{aligned}
$$

and thus

$$
u_{\text {sim }}(\boldsymbol{x}, t)=\frac{Q G_{0}}{\kappa t} e^{-\frac{\mid \boldsymbol{x}^{2}}{4 \kappa t}},
$$

which is the explicit form of the similarity solution we have been seeking. The total mass $Q$ is given by

$$
\int_{\mathbb{R}^{2}} u d^{2} x=4 \pi Q G_{0}
$$


and thus $G_{0}=1 / 4 \pi$.

The typical radius of the drop is

$$
\begin{aligned}
& R=\frac{1}{Q} \int_{\mathbb{R}^{2}}|\mathbf{x}| u_{\operatorname{sim}}(|\mathbf{x}|, t) d^{2} x=\frac{1}{2 \kappa t} \int_{0}^{\infty} r^{2} e^{-\frac{r^{2}}{4 \kappa t}} d r= \\
& \frac{(4 \kappa t)^{3 / 2}}{(2 \kappa t)} \underbrace{\int_{0}^{\infty} \xi^{2} e^{-\xi^{2}} d \xi}_{\sqrt{\pi} / 4}=\sqrt{\pi \kappa} t^{1 / 2},
\end{aligned}
$$

and thus behaves like a power law. According to our reasoning, the power law should be valid for $\delta \ll R \ll L$.

Excursion: What is special about power laws?

Consider

$$
R=M t^{\alpha} \text {. }
$$

The main point is that power laws are scale-invariant, and thus do not specify a particular scale. Any change of t-scale $\left(t_{0}\right)$ can be absorbed into a change of R-scale $\left(R_{0}\right)$ :

$$
R=M t_{0}^{\alpha}\left(t / t_{0}\right)^{\alpha}, R_{0} \equiv t_{0}^{\alpha}
$$

and thus

$$
\tilde{R}=M \tilde{t}^{\alpha},
$$

which is the same as (7), but with $\tilde{R}=R / R_{0}$ and $\tilde{t}=t / t_{0}$.

This property also characterizes power laws: Let

$$
R=f(t) \quad \text { and } \quad R / R_{0}\left(t_{0}\right)=f\left(t / t_{0}\right),
$$

which is the general condition for scale invariance. It follows that

$$
R(t)=f(1) R_{0}(t) \text {, thus } R(t) / R\left(t_{0}\right)=f\left(t / t_{0}\right) / f(1) .
$$

Now differentiate with respect to $t: R^{\prime}(t) / R\left(t_{0}\right)=f^{\prime} /\left(t_{0} f(1)\right)$, and put $t=t_{0}$. This gives

$$
(\ln R)^{\prime}=\frac{f^{\prime}(1)}{f(1)}(\ln t)^{\prime}
$$

and thus $R=R_{0} t^{\alpha}$, where $\alpha=f^{\prime}(1) / f(1)$ and $R_{0}$ is a constant of integration.

Now two more remarks about the results obtained so far are in order:

Remark 1 A crucial ingredient in finding the exponents in the scaling ansatz

$$
u=t^{\alpha} G\left(\frac{r}{t^{\beta}}\right)
$$

(namely $\alpha=-1, \beta=1 / 2$, cf. equation 3 ) was the assumption of regularity in the limits $\delta / \ell \rightarrow 0$ and $L / \ell \rightarrow \infty$. It resulted in rational exponents determined by dimensional analysis alone. If $F(r / \ell, \delta / \ell)$ had not been regular in the limit $\delta / \ell \rightarrow 0$, while keeping with the assumption of self-similarity, the answer would have been:

$$
u=u_{0} F\left(\frac{r}{\ell}\left(\frac{\delta}{\ell}\right)^{\gamma}\right),
$$

with an arbitrary exponent $\gamma$, giving $\beta=(1+\gamma) / 2$. This exponent would have been undetermined and would have to be found by other means. This leads to the following rule of thumb for the classification of self-similarity (Barenblatt): 
(a) Self-similarity of the first kind: the problem is regular as function of the external parameters $\delta, L$. The exponents are rational, determined by dimensional analysis, or symmetry, see below.

(b) Self-similarity of the second kind: the problem is singular as function of $\delta$ and $L$, the exponents are in general irrational, determined by a non-linear eigenvalue problem, see below.

Remark 2: The "dynamical system" description. Assume for simplicity that $u=u(r, t)$ and put $\xi=r / \sqrt{t \kappa}$ as before, as well as logarithmic time $s=\ln t$. Put

$$
u(r, t)=: \frac{1}{t \kappa} W(\xi, s),
$$

so (1) can then be transformed into

$$
\begin{aligned}
& \frac{\partial u}{\partial t}=-\frac{W}{t^{2} \kappa}-\frac{1}{2 t^{2} \kappa} \xi W_{\xi}+\frac{W_{s}}{t^{2}}=\frac{1}{\kappa t^{2}} \frac{1}{\xi}\left(\xi W_{\xi}\right)_{\xi} \\
& \rightarrow \frac{\partial W}{\partial s}=\frac{1}{\kappa}\left[W+\frac{\xi W_{\xi}}{2}+\frac{\left(\xi W_{\xi}\right)_{\xi}}{\xi}\right] .
\end{aligned}
$$

Thus a fixed point $\partial W / \partial s=0$ of this dynamical system corresponds to the similarity solution (5), as it is equivalent to the similarity equation (4). The study of (10) is an extremely useful tool to study the approach to a similarity solution as well as its stability. In addition, the dynamical system (10) is the starting to go beyond fixed-point behavior. For example, in some cases (10) has solutions corresponding to periodic orbits, which corresponds to a generalization of simple similarity solutions such as (8).

The heat equation (1) can of course be solved generally, so the approach to the similarity solution can be demonstrated explicitly, using Green functions. Namely, it is easy to verify that for the special initial condition $u(\mathbf{x}, 0)=Q \delta(\mathbf{x})$ the solution of (1) is (5). This must indeed be so, since (5) was found from the assumption $\delta \rightarrow 0$, which corresponds to an initial $\delta$-function of zero extension.

As usual, a general initial condition can be written as a superposition of $\delta$-functions:

$$
u(\boldsymbol{x}, 0)=\int_{\mathbb{R}^{2}} \delta\left(\boldsymbol{x}-\boldsymbol{x}_{0}\right) u\left(\boldsymbol{x}_{0}, 0\right) d^{2} x_{0} .
$$

Thus from the superposition principle:

$$
u(\boldsymbol{x}, t)=\int_{\mathbb{R}^{2}} \frac{u\left(\boldsymbol{x}_{0}, 0\right)}{4 \pi \kappa t} e^{-\frac{\left|\boldsymbol{x}-\boldsymbol{x}_{0}\right|^{2}}{4 \kappa t}} d^{2} x_{0},
$$

which is formally the most general solution.

To show that (11) always gives (5) in the limit $\delta \rightarrow 0$, we use a Taylor expansion of the kernel around $\boldsymbol{x}$, which is known as a multipole expansion in this context. Namely, let us assume that the initial blob is contained inside a ball of radius $\delta$. The Taylor expansion is

$$
e^{-\frac{\left|\boldsymbol{x}-\boldsymbol{x}_{0}\right|}{4 \kappa t}}=e^{-\frac{|\boldsymbol{x}|}{4 \kappa t}}+\frac{\boldsymbol{x}_{0} \cdot \boldsymbol{x}}{2 \kappa t} e^{-\frac{|\boldsymbol{x}|^{2}}{4 \kappa t}}+O\left(\delta^{2}\right),
$$


which gives

$$
\begin{gathered}
u(\boldsymbol{x}, t)=\underbrace{\frac{Q}{4 \pi \kappa t} e^{-|\boldsymbol{x}| / 4 \kappa t^{2}}}_{u_{\mathrm{sim}}}+\frac{1}{4 \pi \kappa t} \underbrace{\int u\left(\boldsymbol{x}_{0}, 0\right) \boldsymbol{x}_{0} d^{2} \boldsymbol{x}_{0}}_{\boldsymbol{p}} \cdot \boldsymbol{x} \frac{e^{-|\boldsymbol{x}|^{2} / 4 \kappa t}}{2 \kappa t}+0\left(\delta^{2}\right) \\
=u_{\operatorname{sim}}(\boldsymbol{x}, t)+\underbrace{\frac{\boldsymbol{p} \boldsymbol{x}}{8 \pi \kappa^{2} t^{2}}}_{|\boldsymbol{x}| \approx \sqrt{t}, \boldsymbol{p}} e^{-|\boldsymbol{x}|^{2} / 4 \kappa t}+0\left(\delta^{2}\right)=u_{\operatorname{sim}}(\boldsymbol{x}, t)+0\left(\frac{\delta}{\kappa^{2} t^{3 / 2}}, \delta^{2}\right) .
\end{gathered}
$$

Thus we can not only show that the solution converges to (5), but we can also compute corrections showing how this limit is approached.

\subsection{The power of scaling}

Physically, the spreading problem is characterized by the absence of a particular length scale; $\delta<<R<L$, so $\mathrm{R}$ varies by many orders of magnitude.

Crucial Properties of scaling laws are:

(a) universality (independence of initial condition). n.b.: in the linear case the solution cannot be completely universal, as it is still depends on Q, set by the initial condition.

(b) scaling form $u=t^{\alpha} G\left(\frac{r}{t^{\beta}}\right)$

(c) power laws, either (i) rational (first kind) or (ii) irrational (second kind)

But beware: there are examples where one or all of these properties break down: one often need to understand more about the problem. A particular case where things are more complicated is

Example 1 snowflakes, see Fig.2. Not only does the form of the snowflake seem to depend on initial condition, it even depends on the (sixfold) symmetry of the crystal, although lattice constant $\simeq 1 n m<<<R$ ! The reason must lie in instabilities, which cause small perturbations to get amplified, as it often occurs in far-from-equilibrium processes. Implicit in our analysis of (1) was the fact that certain microscopic parameters can be eliminated from the problem, an argument that cannot readily be made for the snow flake. However, an example where simple dimensional analysis provides deep insight into an extremely complicated problem is

Example 2 turbulent diffusion, see Fig.3. The amount of stirring (turbulence) is characterized by the energy input $\varepsilon$ per time and mass

$$
\varepsilon=\frac{\text { energy }}{\text { time }}=\frac{\text { power }}{\text { mass }}, \quad[\varepsilon]=\frac{\mathrm{cm}^{2}}{s^{2} \cdot s}=\frac{\mathrm{cm}^{2}}{s^{3}} .
$$

We have

$$
R(t)=f(t, \varepsilon, \delta, \nu),
$$

where $\delta$ is the initial size and $\nu$ the kinematic viscosity of the fluid in which the spreading occurs, $[\nu]=\mathrm{cm}^{2} / \mathrm{s}$. 


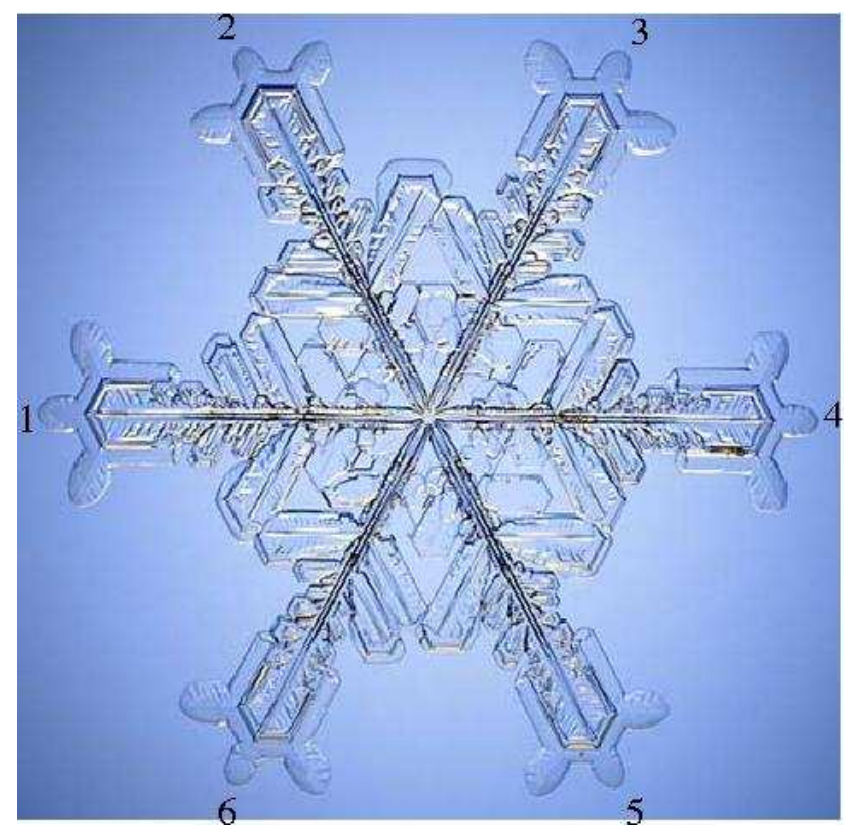

Figure 2: A naturally grown snowflake, from Libbrecht and Rasmussen (2003). It exhibits the six-fold symmetry of an ice crystal on a macroscopic scale.

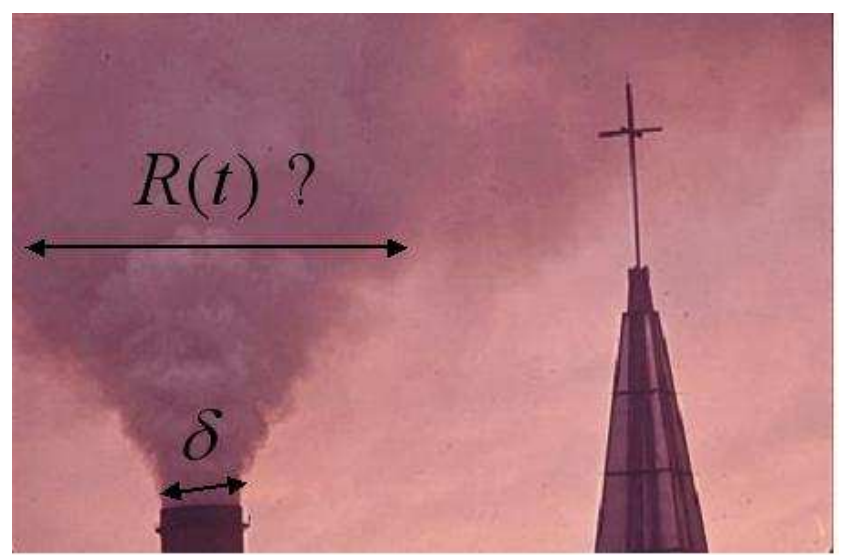

Figure 3: Smoke spreading in a turbulent environment 
If the transport is governed by turbulent motion alone, independent of fluid viscosity or initial condition, we have $R(t)=f(t, \varepsilon)$. The only way to combine $\varepsilon$ and $t$ to get a length is $\left[\varepsilon t^{3}\right]=\mathrm{cm}^{2}$, thus $R=A\left(\varepsilon t^{3}\right)^{1 / 2}$, much faster than $t^{1 / 2}$, i.e. by diffusion.

Diversion: the idea of dimensional analysis is that no law of nature can depend on the units we give to the fundamental quantities of mass, length, time, and charge. Mathematically, this leads to a certain invariance under scale transformations (for details, see Birkhoff (1950)). More formally:

(i) Assume that there are $n$ fundamental units $q_{i}, i=1 \ldots n$, which can be changed by multiplying with a scale factor $\alpha_{i}: T\left(q_{i}\right)=\alpha_{i} q_{i}$.

(ii) The "derived" quantities $Q_{j}$ (such as density, velocity etc) then transform like $T\left(Q_{j}\right)=Q_{j} \Pi_{i=1}^{n} \alpha_{i}^{a_{j i}}$. The exponents $a_{j i}$ are called the dimensions of $Q_{j}$. Now any physical law $f\left(Q_{1}, \ldots Q_{k}\right)=0$ must be $\underline{\text { unit free, }}$, i.e. invariant under all transformations $T$.

Example 3 Navier-Stokes equation

$$
\frac{\partial \boldsymbol{u}}{\partial t}+(\boldsymbol{u} \nabla) \boldsymbol{u}=-\frac{1}{\rho} \nabla p+\nu \Delta \boldsymbol{u}
$$

All terms transform like $T(\partial \boldsymbol{u} / \partial t)=\mathrm{cm} / \mathrm{s}^{2}$, thus $T(N S)=N S$. This means all physical laws derived from $(N S)$ must have the same invariance.

The statement that permits to simplify $f\left(Q_{1}, \ldots, Q_{k}\right)=0$ given that $T f=$ 0 is known as the "Buckingham $\Pi$-Theorem". To formulate it, we have to make a technical assumption. Let $m \leqslant n$ be the rank of the $a_{i j}$ - matrix defined by $T\left(Q_{j}\right), j=1, \ldots k$.

Then $f\left(Q_{1}, \ldots, Q_{k}\right)=0$ is equivalent to $\phi\left(\Pi_{1}, \ldots, \Pi_{k-m}\right)=0$, where the $\Pi_{\ell}$ are dimensionless. Thus one obtains a reduction of the number of variables by $m$ without loss of information! The $\Pi_{\ell}$ correspond exactly to the expression $r / \sqrt{\kappa t}$ above and thus help to find power laws.

Applications include all systems with many length scales

(a) continuum mechanics: singularities (see below), turbulence, explosions, cracks

(b) critical phenomena (systems near phase transitions), where correlation length $\rightarrow \infty$

(c) elementary particles (where the energy becomes very large)

(d) cosmology: black holes, etc.

Generalizations of scaling behavior are logarithms (breaking of scale invariance, see below), and discrete self-similarity, where solutions are only selfsimilar on a discrete set.

\subsection{Drop pinch-off: a non-linear example}

A drop falls from a faucet of radius $r_{0}$ and separates: this must be a singularity of the equations of motion. One way of seeing this is that after the singularity (separation) the drop lives on as an independent entity. Thus the mathematical 


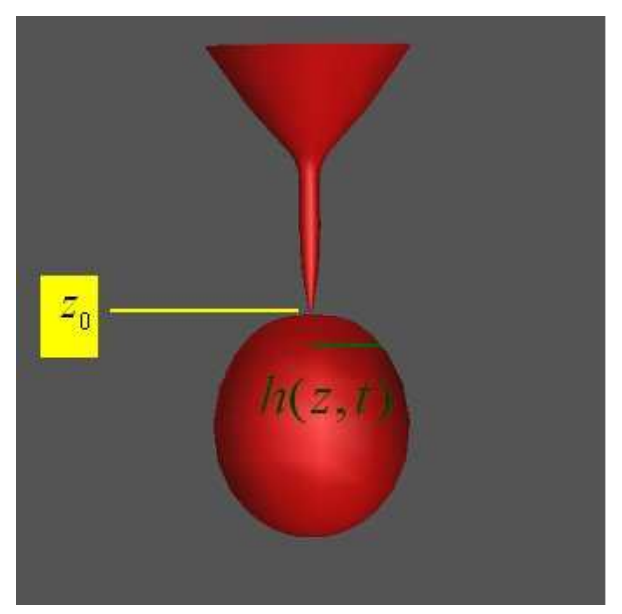

Figure 4: A drop separates from a faucet. At a time $t_{0}$ and position $z_{0}$ the radius of the fluid column goes to zero.

problem changes its character at the singularity: what used to be one single equation describing a single drop of fluid, has now split into two independent equations. Another way of looking at it is that the radius of the fluid neck going to zero locally corresponds to the production of arbitrarily small scales in finite time, and thus to a singularity, i.e. a place where functions are no longer smooth. This will be evident from the similarity description given below.

How does the profile look near the breakup point $z_{0}, t_{0}$ ? The external parameters are $\rho$ (density), $\nu$ (kinematic viscosity), $\gamma$ (surface tension), $g$ (acceleration of gravity), and $r_{0}$ (nozzle radius). We are interested in the limit $\Delta t=t_{0}-t$ and $\Delta z=z-z_{0}$ small. Thus for $\Delta t \rightarrow 0$, a typical length scale characteristic for breakup is $\ell_{t}=\sqrt{\nu \Delta t}$, keeping in mind that

$$
[\nu]=\frac{\mathrm{cm}^{2},}{s},[\gamma / \rho]=\frac{\mathrm{cm}^{3}}{s^{2}},[g]=\frac{\mathrm{cm}}{s^{2}} .
$$

Thus using dimensional analysis,

$$
h=f\left(\Delta z, \Delta t, s, \nu, \gamma, g, r_{0}\right)
$$

can be reduced to

$$
h=\ell_{t} \bar{f}\left(\frac{\Delta z}{\ell_{t}}, \frac{\ell_{\nu}}{\ell_{t}}, \frac{\ell_{c}}{\ell_{t}}, \frac{r_{0}}{\ell_{t}}\right),
$$

where $\ell_{\nu}=\nu^{2} \gamma / \rho$ is an intrinsic viscous length scale, and $\ell_{c}=\sqrt{\gamma / \rho g}$ is the (macroscopic) capillary length (typically $1 \mathrm{~mm}$ ).

As argued before, in the limit $\ell_{t} \rightarrow 0$ the scales $\ell_{c}$ and $r_{0}$ shouldn't matter, so $\bar{f}$ should have a finite limit for $\ell_{c} / \ell_{t} \rightarrow \infty, r / \ell_{t} \rightarrow \infty$. But one still has

$$
h=\ell_{t} \bar{f}\left(\frac{\Delta z}{\ell_{t}}, \frac{\ell_{\nu}}{\ell_{t}}\right), \quad v=\bar{g}\left(\frac{\Delta z}{\ell_{t}}, \frac{\ell_{\nu}}{\ell_{t}}\right),
$$

where $v(z, t)$ is the average velocity in the $z$-direction.

Thus a (possible) similarity form cannot be found from dimensional analysis alone. However for the special case $\nu=0$ (inviscid fluid), progress is possible. 
For example, for water $\ell_{\nu} \approx 10 \mathrm{~nm}$, so as long as $h_{\min }>>\ell_{\nu}$ we hope to effectively have $\ell_{\nu}=0$. Assuming that this limit is indeed regular, we can redo our dimensional analysis, using $\ell_{i n}=\left(\widetilde{\gamma} \Delta t^{2}\right)^{1 / 3}$ as our length scale noting that $[\widetilde{\gamma}]=[\gamma / \rho]=\mathrm{cm}^{3} / \mathrm{s}^{2}$. The only possible scaling form is then

$$
h=\ell_{\text {in }} H_{\text {in }}\left(\frac{\Delta z}{\ell_{\text {in }}}\right), \quad v=\frac{\ell_{\text {in }}}{\Delta t} V_{\text {in }}\left(\frac{\Delta z}{\ell_{\text {in }}}\right) .
$$

Some remarkable results follow:

$$
h_{\min }=a_{i n}\left(\gamma / \rho \Delta t^{2}\right)^{1 / 3}, \text { where } \quad a_{\text {in }} \approx 0.7
$$

from experiment and numerical simulations. Likewise, the entire scaling function $H_{i n}$, which gives the form of the neck near pinch-off, is completely universal and can in principle be calculated!

In the more general case of finite $\ell_{\nu}$, one has to look at the equation underlying the dynamics. With some simplifications, they are

$$
\begin{gathered}
\frac{\partial h^{2}}{\partial t}+\left(h^{2} v\right)_{z}=0 \quad \text { (mass conservation) } \\
\frac{\partial v}{\partial t}+v v_{z}=-\tilde{\gamma}\left(\frac{1}{h}\right)_{z}+3 \nu \frac{\left(v_{z} h^{2}\right)_{z}}{h^{2}}+g . \quad \text { (force balance). }
\end{gathered}
$$

This set of equations has a special symmetry: if one substitutes $\tilde{h}=h / \tilde{\gamma}$, then $\nu$ and $g$ are the only parameters left:

$$
\begin{aligned}
& \frac{\partial \tilde{h}^{2}}{\partial t}+\left(\tilde{h}^{2} v\right)_{z}=0 \\
& \frac{\partial v}{\partial t}+v v_{z}=-\left(\frac{1}{\tilde{h}}\right)_{z}+3 \nu \frac{\left(v_{z} \tilde{h}^{2}\right)_{z}}{\tilde{h}^{2}}+g .
\end{aligned}
$$

Thus if one writes the dimensionally correct expressions for $\tilde{h}$, which has dimensions $[\tilde{h}]=s^{2} / \mathrm{cm}^{2}$, then $\ell_{\nu}=\nu^{2} / \tilde{\gamma}$ cannot appear, since it contains $\tilde{\gamma}$. But this means (13) can in fact be written

$$
\tilde{h}=\frac{(\Delta t)^{2}}{\ell_{t}^{2}} H\left(\frac{\Delta z}{\ell_{t}}\right), \quad v=\frac{\ell_{t}}{\Delta t} V\left(\frac{\Delta z}{\ell_{t}}\right) .
$$

Since $\tilde{\gamma}$ is just a constant, the similarity forms for the original variables $h, v$ are

$$
h(z, t)=t^{\prime} \phi\left(\frac{z^{\prime}}{t^{\prime / 2}}\right), \quad v(z, t)=t^{\prime-1 / 2} \psi\left(\frac{z^{\prime}}{t^{1 / 2}}\right),
$$

where $z^{\prime}=\Delta z / \ell_{\nu}, t^{\prime}=\Delta t / t_{\nu}$ are convenient dimensionless length and time scales and $t_{\nu}=\nu^{3} \rho^{2} / \gamma^{2}$. Again, it is implicit in the analysis that $\phi, \psi$ are universal. For example,

$$
h_{\min }=a \frac{\gamma}{\rho \nu} \Delta t, \quad a \approx 0,03 .
$$

To compute $\phi, \psi$, one inserts the similarity form into (15),(16). Namely, it is easily confirmed that in the force balance of (16)

$$
\frac{\partial v}{\partial t} \sim v v_{z} \sim\left(\frac{1}{h}\right)_{z} \sim \frac{\left(v_{z} h^{2}\right)_{z}}{h^{2}} \sim \Delta t^{-3 / 2}
$$


i.e. these terms blow up with the same power for $\Delta t \rightarrow 0$. By contrast, in (16), the contribution from gravity scales like $g \sim(\Delta t)^{0}$, and will drop out in the limit $\Delta t \rightarrow 0$. This is the important concept of dominant balance, which can also be used to show that all terms present in the hydrodynamic description of pinch-off are subdominant relative to the ones kept in the "slender-jet" description (15),(16). The dominant balance means that gravity is not important for pinch-off, as was implicit in our earlier assumption that $\ell_{c}$ drops out from (12) in the limit $\Delta t \rightarrow 0$.

The similarity equations for $\phi, \psi$, as obtained from (15) and (16), are

$$
\begin{aligned}
& -2 \phi^{2}+\xi \phi \phi^{\prime}+\left(\phi^{2} \psi\right)^{\prime}=0 \\
& \frac{\psi}{2}+\frac{\xi \psi^{\prime}}{2}+\psi \psi^{\prime}=-\left(\frac{1}{\phi}\right)^{\prime}+3 \frac{\left(\psi^{\prime} \phi^{2}\right)^{\prime}}{\phi^{2}},
\end{aligned}
$$

where $\xi=z^{\prime} / t^{\prime 1 / 2}$. This is an O.D.E. to be solved for $\phi, \psi$; it can be solved uniquely using proper boundary constrictions, which we do not discuss here.

Finally, it is natural to look at the other limiting case where $\ell_{\nu}>>r_{0}$, much larger then all scales in the problem. Formally, this is best incorporated by putting $\rho=0$, so there is no inertia in the problem. This leaves $\gamma$ and $\eta=\nu \cdot \rho$ as the only parameters. Now $[\gamma / \eta]=\mathrm{cm} / s$ and thus $\ell_{v i s}=v_{\eta} \Delta t\left(v_{\eta}=\gamma / \eta\right)$ is a convenient local length scale. Following the same logic as before one obtains:

$$
h=\ell_{\mathrm{vis}} H_{\mathrm{vis}}\left(\frac{\Delta z}{\ell_{\mathrm{vis}}}\right), \quad v=\frac{\ell_{\mathrm{vis}}}{\Delta t} V_{\mathrm{vis}}\left(\frac{\Delta z}{\ell_{\mathrm{vis}}}\right) .
$$

All seems well, but the result is wrong!

The reason is that the limit $r_{0} / \ell_{\text {vis }} \rightarrow \infty$ is not regular, signaling selfsimilarity of the second kind. Namely, for $\rho=0$ (16) reads

$$
\begin{aligned}
v_{\eta}\left(\frac{1}{h}\right)_{z} & =3 \frac{\left(v_{z} h^{2}\right)_{z}}{h^{2}} \mid \cdot h^{2} \quad \text { and so } \\
-h & =\frac{3}{v_{\eta}} v_{z} h^{2}-C(t),
\end{aligned}
$$

or

$$
\frac{3 v(z)}{v_{\eta}}=-\int_{0}^{z}\left(\frac{C^{\prime}}{h^{2}}+\frac{1}{h}\right) d z .
$$

Thus $v$ is determined by $h$ alone!

The equations (15),(19) remain invariant under the transformation $z=$ $a \tilde{z}, v=a \tilde{v}$, hence $H_{v i s}, V_{v i s}$ cannot possibly be universal! Instead, the local solution must depend in some way on initial conditions, i.e. $r_{0}$. Let us assume that solutions remain self-similar, but depend on both combinations $\Delta z / \ell_{\text {vis }}$ and $r_{0} / \ell_{\text {vis }}$. Then using the invariance under the above transformation we obtain

$$
h=\ell_{\mathrm{vis}} H_{\mathrm{vis}}\left(\frac{\Delta z}{\ell_{\mathrm{vis}}}\left(\frac{\ell_{\mathrm{vis}}}{r_{0}}\right)^{1-\beta}\right), \quad v=u_{\eta}\left(\frac{\ell_{\mathrm{vis}}}{r_{0}}\right)^{\beta-1} V_{\mathrm{vis}}\left(\frac{\Delta z}{\ell_{\mathrm{vis}}}\left(\frac{\ell_{\mathrm{vis}}}{r_{0}}\right)^{1-\beta}\right) .
$$

To find the scaling exponent $\beta$, dimensional analysis evidently is not enough. One really has to use the (viscous) equation (20) together with (15). 
To solve this viscous system of equations, we use a clever transformation, employing a particle marker as the spatial variable (Lagrangian transformation). Let $\alpha$ mark a fluid volume in the form of a slice $h^{2}(\alpha t) d z=d \alpha$. Then $z_{\alpha}=1 / h^{2}$ and $z_{t}=v$ (since $\alpha$ marks a material object). Here $z(\alpha, t)$ is the position of the volume $\alpha$ at time $t$. The first transformation incorporates volume conservation. Equation (19) transforms to (using $\partial_{z}=z_{\alpha}^{-1} \partial_{\alpha} \rightarrow v_{z}=z_{\alpha}^{-1} \partial_{\alpha} z_{t}$ )

$$
\begin{aligned}
& \frac{1}{z_{\alpha}^{1 / 2}}+\frac{3}{v_{\eta}} \frac{z_{\alpha} t}{z_{\alpha}^{2}}=C(t), \text { which, with } \\
& H(\alpha, t)=\frac{1}{\sqrt{z_{\alpha}}} \text { is } H-\frac{6}{v_{\eta}} H_{t} H=C(t) .
\end{aligned}
$$

The last equation no longer contains a spatial derivative! Introducing the dimensionless variables $t^{\prime}=v_{\eta} \Delta t / r_{0}, \alpha^{\prime}=\alpha / r_{0}^{3}$, we look for the similarity description

$$
H=t^{\prime} \chi\left(\frac{\alpha^{\prime}}{t^{2+\beta}}\right), \quad C=t^{\prime} \bar{C}
$$

where $\zeta=\alpha^{\prime} / t^{2+\beta}$.

The similarity equation is then

$$
\bar{C}=\chi+6 \chi^{2}-6(2+\beta) \zeta \chi \chi^{\prime} .
$$

The constant can be determined using a constraint implicit in $z_{\alpha}=1 / H^{2}$, and hence $1 / \chi^{2}=F_{\zeta}$, where $F(\zeta)$ is some function. Dividing by $\chi^{4}$ :

and thus

$$
\bar{C} / \chi^{4}=1 / \chi^{3}+\underbrace{\underbrace{6 F_{\zeta}}_{\left[F / \chi^{2}\right.}-6(2+\beta) \underbrace{\zeta \chi^{\prime} / \chi^{3}}}_{\left[F+3(2+\beta)\left(\zeta F_{\zeta}-F\right)\right]_{\zeta}}
$$

$$
\bar{C} \int_{-\infty}^{\infty} \frac{d \zeta}{\chi^{4}}=\int_{-\infty}^{\infty} \frac{d \zeta}{\chi^{3}}
$$

Integrating (23) we get

$$
\frac{1}{6(2+\beta)} \ln \left|\frac{\zeta}{\bar{\zeta}}\right|=\int_{\bar{\chi}}^{\chi} \frac{\zeta d \zeta}{6 \zeta^{2}+\zeta-\bar{C}}, \bar{\chi}=\chi(\bar{\zeta}) .
$$

Locally (near the minimum) $\chi$ looks like $\chi=\chi_{0}+\zeta^{2}+O\left(\zeta^{4}\right)+\ldots$

Here we used the fact that the $\zeta$-scale is arbitrary, so the quadratic coefficient was normalized to unity. Performing the integral gives

$$
\left|\frac{\zeta}{\bar{\zeta}}\right|^{\frac{2}{2+\beta}}=\left|\frac{6 \chi^{2}-\chi-\bar{C}}{6 \bar{\chi}^{2}-\bar{\chi}-\bar{C}}\left(\frac{(\Gamma+1+12 \chi)(\Gamma-1-12 \bar{\chi})}{(\Gamma-1-12 \chi)(\Gamma+1+12 \bar{\chi})}\right)^{\frac{1}{\Gamma}}\right|
$$

where $\Gamma=\sqrt{1+24 \bar{C}}$. Since $\bar{\chi} \rightarrow \chi_{0}$ as $\bar{\zeta} \rightarrow 0$ one must have $6 \chi_{0}^{2}-\chi_{0}-\bar{C}=0$, and since powers of $\zeta$ must match for $\zeta \rightarrow 0$ we get $-\frac{2}{2+\beta}=-2+\frac{2}{\Gamma}$. Combining both equations results in

$$
\chi_{0}=\frac{1}{12(1+\beta)}, \quad \bar{C}=\frac{3+2 \beta}{24(1+\beta)^{2}} .
$$


Now (24) can be understood as an equation for $\beta$ ! By changing the variable of integration from $\zeta$ to $\chi$, the integrals can be computed in the following three steps:

(i) Equation (23) can be solved to give

$$
\zeta=\left[\frac{6(1+\beta)}{2+\beta}\left(\chi+\frac{3+2 \beta}{12(1+\beta)}\right)\right]^{\frac{3+2 \beta}{2}}\left(\chi-\chi_{0}\right)
$$

(ii) One has

$$
\int_{-\infty}^{\infty} \frac{d \zeta}{\chi^{i}}=2 \int_{\chi_{0}}^{\infty} \frac{d \chi}{\chi^{i} \chi^{\prime}}, \text { where } \chi^{\prime}=\frac{6 \chi^{2}+\chi-\bar{C}}{6(2+\beta) \chi \zeta}
$$

from (23), and $\zeta$ is expressible through $\chi$ by $(26)$.

(iii) Evaluating both sides of (24) as integrals over $\chi$ gives

$$
\frac{(1-\beta)(3+2 \beta)}{(1+\beta)(3-2 \beta)}=\frac{F\left(-\frac{1}{2}-\beta, 1-\beta ; 3 / 2-\beta ; 3-2 \beta\right)}{F\left(-\frac{1}{2}-\beta, 2-\beta ; 5 / 2-\beta ;-3-2 \beta\right)} \text {. }
$$

Solving this last equation gives $\beta=0,1748717 \ldots$

Thus the nonlinear eigenvalue problem $(23),(24)$ indeed leads to a nontrivial, irrational exponent which is typical for self-similarity of the second kind. One then finds $\chi_{0} \approx 0,071$, thus

$$
h_{\min }=a_{v i s} \frac{\gamma}{\rho \nu} \Delta t, a_{v i s}=0,0709 .
$$

Again, the minimum of $H_{v i s}$ is universal, however the entire function is not, its width depends on initial conditions, as shown earlier.

\subsection{Logarithms: lavalamp dynamics}

A novel phenomenon occurs if dripping takes place inside an ambient fluid: the simplest case is that of the viscosities of both fluids being equal. The interface then behaves like an elastic membrane of surface tension $\gamma$. This means each point of the interface exerts a point force $\mathbf{n} \cdot$ curvature $\approx \mathbf{n} / h$ if the profile is slender. The total velocity can be written as an integral over all point forces:

$$
v(z)=\frac{1}{4} \int_{\text {drop }} \frac{h_{z^{\prime}}\left(z^{\prime}\right)}{\sqrt{h^{2}\left(z^{\prime}\right)+\left(z-z^{\prime}\right)^{2}}} d z^{\prime},
$$

having simplified the expression by assuming $h_{z}(z)<<1$.

This is to be coupled to the usual mass balance (15). Dimensionally, one would once more expect a local solution of the form

$$
h(z, t)=\ell_{\mathrm{vis}} H_{\mathrm{out}}\left(\frac{\Delta z}{\ell_{\mathrm{vis}}}\right), \quad \ell_{\mathrm{vis}}=v_{\eta} \Delta t .
$$


Now as $\ell_{\text {vis }} \rightarrow 0$ and $\Delta z$ is finite, one expects $h\left(\Delta z, t_{0}\right)$ to remain finite. But this means $H_{\text {out }}$ must be linear at infinity

$$
H_{\text {out }}(\xi) \rightarrow\left\{\begin{array}{ll}
H_{+} \xi & \xi \rightarrow \infty \\
H_{-} \xi & \xi \rightarrow-\infty .
\end{array} \quad \xi=\frac{\Delta z}{\ell_{\text {vis }}}\right.
$$

In similarity variables, (28) has the form

$$
V_{\text {out }}(\xi)=\frac{1}{4} \int_{-z_{b} / \ell_{\text {vis }}}^{z_{b / \ell_{\mathrm{vis}}}} \underbrace{\frac{H_{\text {out }}^{\prime}\left(\xi^{\prime}\right)}{\sqrt{H_{\text {out }}^{2}+\left(\xi-\xi^{\prime}\right)^{2}}}}_{\text {arg }} d \xi^{\prime}
$$

But if $H_{\text {out }}$ is linear, the argument behaves like

$$
\arg \left(\xi^{\prime}\right) \rightarrow \begin{cases}\left(H_{+} / \sqrt{H_{+}^{2}+1}\right) \xi, & \xi \rightarrow \infty \\ \left(H_{-} / \sqrt{H_{-}^{2}+1}\right) \xi, & \xi \rightarrow-\infty\end{cases}
$$

which results in a divergent integral!

This can be fixed using a simple trick:

$$
h=\ell_{\mathrm{vis}} H_{\text {out }}(\xi), \quad \xi=\frac{\Delta z}{\ell_{\mathrm{vis}}}+b \ln \Delta t,
$$

i.e. moving the $z$-axis at a speed which depends logarithmically on time! With this modification, the mass balance becomes

$$
-H_{\text {out }}+H_{\text {out }}^{\prime}\left(\xi+V_{\text {out }}-b \ln \Delta t\right)=H_{\text {out }} V_{\text {out }}^{\prime} / 2 .
$$

Now we choose $b$ such that the logarithmic singularity cancels, namely

$$
\frac{1}{4} \int_{-z_{b} / \ell_{\mathrm{vis}}}^{z_{b} / \ell_{\mathrm{vis}}} \frac{H_{\text {out }}^{\prime}\left(\xi^{\prime}\right)}{\sqrt{H_{\text {out }}^{2}+\left(\xi-\xi^{\prime}\right)^{2}}} d \xi^{\prime}-b \ln \left(\ell_{\mathrm{vis}}\right)
$$

finite for $\ell_{\text {vis }} \rightarrow 0$. This is achieved by putting

$$
b=\frac{1}{4}\left[-\frac{H_{+}}{\sqrt{H_{+}^{2}+1}}+\frac{H_{-}}{\sqrt{H_{-}^{2}+1}}\right] .
$$

Thus defining

$$
V_{\text {fin }}(\xi)=\lim _{\Lambda \rightarrow \infty} \frac{1}{4} \int_{-\Lambda}^{\Lambda} \frac{H_{\text {out }}^{\prime}}{\sqrt{H_{\text {out }}^{2}+\left(\xi-\xi^{\prime}\right)^{2}}} d \xi^{\prime}+b \ln \Lambda
$$

the similarity equation

$$
-H_{\text {out }}+H_{\text {out }}^{\prime}\left(\xi+V_{\text {fin }}-\xi_{0}\right)=H_{\text {out }} V_{\text {fin }}^{\prime} / 2
$$

is finite, and $\xi_{0}$ is an arbitrary constant. 
The numerical solution of this integro-differential equation gives

$$
h_{\min }=a_{\text {out }} v_{\eta} \Delta t, \quad \text { where } \quad a_{\text {out }}=0,0335 \quad \text { and } \quad H_{+}=4,81 ; H_{-}=-0,105 .
$$

These constants are universal, the whole similarity profile $H_{\text {out }}$, however, is not, for the following reason: whereas in previous examples the singular motion was self-similar,

$$
h(z, t)=\ell_{\text {vis }} H_{\text {out }}\left(\frac{\Delta z}{\ell_{\text {vis }}}+b \ln \Delta t\right)
$$

is not:a change in $\ell_{\text {vis }}$ cannot be absorbed into rescaling of the axes.

\subsection{Nanojets: fluctuations}

On small scales, thermal motion becomes ever more important. Fluid elements behave like Brownian particles, and lead to increasing fluctuations. To estimate fluctuations of an interface, one constructs the thermal length scale $\ell_{T}=\sqrt{k_{B} T / \gamma}$, comparing thermal and surface tension energies; it is typically $1 \mathrm{~nm}$. This means a liquid bridge "wobbles" increasingly as it pinches off.

This behavior is indeed seen in simulations (microscopic "MD" simulations of individual atoms (Moseler and Landman (2000))) and in experiments. The experiments (Aarts et al. (2004)) use a colloid-polymer mixture, which separates into two phases, with very small $(\gamma \approx 0,2 \mu N / m)$ surface tension between them. Thus $\ell_{T}$ is $\approx 10 \mu \mathrm{m}$, and fluctuations are macroscopically observable.

In particular, one important observation is made: instead of $h_{\min } \propto \Delta t$, pinch-off occurs much more abruptly: typically $h_{\min } \propto \Delta t^{0.4}$. How do fluctuations succeed in driving the system toward a singularity more rapidly? To answer this question, fluctuations must be added to the dynamics. To avoid complications, we introduce a radical simplification: the entire bridge is treated as if it were just a single particle of mass $m_{\mathrm{eff}}=m(h)$. Of course, this mass will be smaller if the bridge radius is small, see Fig. 5.

The simplest possible set of equations is

$$
\dot{h}=v \quad m(h) \dot{v}=\xi,
$$

where $\xi(t)$ is Gaussian white noise. This means a discrete version is:

$$
P\left(\xi_{j}\right)=e^{-\xi_{j}^{2} / 2},<\xi_{i} \xi_{j}>=\delta_{i j} .
$$

The probability of finding an entire sequence is thus

$$
\omega\left\{\xi_{i}\right\}=\exp \left\{-\frac{1}{2} \sum_{i} \xi_{i}^{2}\right\} .
$$

For a continuous path $h(t), v(t)$ we thus have the probability

$$
\omega\{h(t), v(t)\}=e^{-S}, \quad S=\frac{1}{2} \int(m \dot{v})^{2} d t .
$$

Formally, any path $h(t)$ is possible, but each is assigned a different probability. A way to characterize the typical behavior is to look for the most probable 


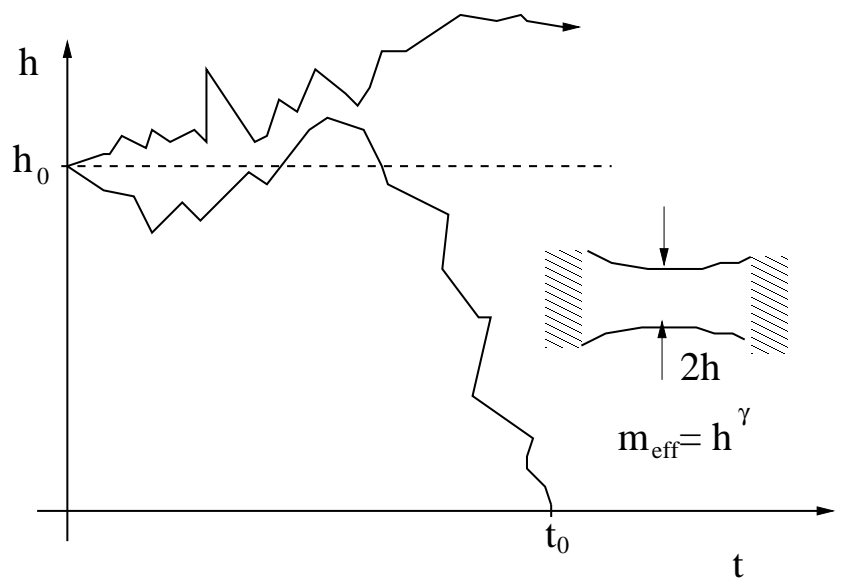

Figure 5: A very simple model of a "nanobridge". The only variable is the minimum radius $h$. We are only interested in paths which lead to pinch-off in some finite time $t_{0}$.

path. Such an optimal path is $h=h_{0}, v=0$, since this makes $S=0$, which is an absolute minimum.

However, this ignores the fact that paths do not last forever, but rather end at different singular times $t_{0}$. It is thus a more well-defined questions to fix $t_{0}$ and to ask for the most probable path that begins at $\left(0, h_{0}\right)$, and ends at $\left(t_{0}, 0\right)$ ! This corresponds to the classical Euler-Lagrange problem of finding the minimum of $S$ with fixed endpoints. The equation $\dot{h}=v$ has to be built in as a constraint:

$$
S=\int \frac{m^{2}(h)}{2} \dot{v}^{2}+\tilde{h}(\dot{h}-v) d t .
$$

The Euler-Lagrange equations read

$$
\begin{gathered}
\frac{d}{d t} \frac{\partial S}{\partial \dot{v}}-\frac{\partial S}{\partial v}=\frac{d}{d t} m^{2} \dot{v}+\tilde{h}=\underset{\uparrow \text { "force" }}{0} . \\
\frac{d}{d t} \frac{\partial S}{\partial \dot{h}}-\frac{\partial S}{\partial h}=\dot{\tilde{h}}-\gamma h^{2 \gamma-1} \dot{v}^{2}=0 .
\end{gathered}
$$

The intriguing feature is that an effective force $\tilde{h}$ has emerged, which could drive the bridge toward pinch-off.

It is convenient to pass to a "Hamiltonian" description with momentum variables $\tilde{h}=\partial S / \partial \dot{h}$ and $\tilde{v}=\partial S / \partial \dot{v}=m^{2} \dot{v}$. This leads to the four equations

$$
\begin{aligned}
& \dot{h}=v \quad \dot{\tilde{h}}=\gamma \tilde{v}^{2} / h^{2 \gamma+1} \\
& \dot{v}=\tilde{v} / h^{2 \gamma} \quad \dot{\tilde{v}}=-\tilde{h},
\end{aligned}
$$

which are to be solved with boundary values at $t=0$ and $t_{0}$. Instead of solving (34) completely, we are just interested in scaling solutions for $\Delta t=t_{0}-t \rightarrow 0$ :

$$
\begin{array}{ll}
h=A_{1} \Delta t^{\alpha_{1}} & \tilde{h}=A_{3} \Delta t^{\alpha_{3}} \\
v=A_{2} \Delta t^{\alpha_{2}} & \tilde{v}=A_{4} \Delta t^{\alpha_{4}} .
\end{array}
$$


Inserting this into (34), there are non-trivial solutions for the set of exponents. In particular

$$
\alpha_{1}=\frac{4}{2 \gamma+2} .
$$

Thus indeed if $\gamma>1$ (the mass decreases sufficiently rapidly with $h$ ) $\alpha_{1}<1$, and pinch-off is faster than linear! 


\section{Bibliography}

D.G.A.L. Aarts, M. Schmidt, and H.N.W. Lekkerkerker. Science, 304:847, 2004.

G.I. Barenblatt. Scaling, Self-Similarity, and Intermediate Asymptotics. Cambridge, 1996.

G. Birkhoff. Hydrodynamics: A study in logic, fact, and similitude. Princeton, 1950.

K. Libbrecht and P. Rasmussen. The Snowflake: Winter's secret beauty. Voyageur Press, 2003.

M. Moseler and U. Landman. Science, 289:1165, 2000. 